\title{
Quantum corrections to dynamical holographic thermalization: entanglement entropy and other non-local observables
}

\section{Walter H. Baron and Martin Schvellinger}

IFLP-CCT-La Plata, CONICET and Departamento de Física, Universidad Nacional de La Plata, Calle 49 y 115, C.C. 67, (1900) La Plata, Buenos Aires, Argentina

E-mail: wbaron@fisica.unlp.edu.ar, martin@fisica.unlp.edu.ar

ABSTRACT: We investigate the thermalization time scale in the planar limit of the $\mathrm{SU}(N)$ $\mathcal{N}=4$ SYM plasma at strong yet finite 't Hooft coupling by considering its supergravity dual description, including the full $\mathcal{O}\left(\alpha^{\prime 3}\right)$ type IIB string theory corrections. We also discuss on the effects of the leading non-planar corrections. We use extended geometric probes in the bulk which are dual to different non-local observables in the $\mathcal{N}=4 \mathrm{SYM}$ theory. This is carried out within the framework of dynamical holographic thermalization.

KEYwORDs: AdS-CFT Correspondence, Holography and quark-gluon plasmas

ARXIV EPRINT: 1305.2237 


\section{Contents}

1 Introduction and motivation $\quad 1$

2 Leading type IIB string theory corrections 4

3 Quantum corrections to holographic thermalization $\quad 6$

3.1 A collapsing thin shell in Anti de Sitter space 6

$\begin{array}{lll}3.2 & \text { Probing thermalization: non-local observables } & 7\end{array}$

4 Results and discussion $\quad 11$

5 Conclusions $\quad 13$

\section{Introduction and motivation}

Heavy-ion collision experiments at the Relativistic Heavy Ion Collider (RHIC) and the Large Hadron Collider (LHC) lead to the formation of a state of QCD matter known as quark-gluon plasma (QGP). It has been shown that the hydrodynamical behavior of such a system is compatible with low values of the viscosity. This is an indication that the referred QGP is strongly coupled. Therefore, perturbative quantum field theory methods are not suitable to investigate this plasma. This is where the gauge/string duality enters, since it allows one to describe properties of a strongly coupled gauge theory in terms of its dual gravitational description. In particular, a holographic dual pair which has been very useful to understand important results of heavy-ion collider physics is given by the planar limit of the $\mathrm{SU}(N) \mathcal{N}=4$ supersymmetric Yang-Mills theory (SYM), and by type IIB supergravity theory on the anti-de Sitter Schwarzschild black hole times a $S^{5}$ background. Motivated by QCD and $\mathrm{SU}(N) \mathcal{N}=4 \mathrm{SYM}$ lattice calculations, it has been argued (see for instance [1] and references therein) that for QGP equilibrium temperatures which are just above the QCD deconfinement temperature, several properties of both gauge theories behave in a similar way, even quantitatively. This supports the idea of using $\mathrm{SU}(N) \mathcal{N}=4$ SYM theory, which is much more symmetric and therefore a much easier theory to work with than the strongly coupled regime of QCD, in terms of its dual string theory model at finite temperature in order to describe the strongly coupled plasma.

In an actual collision of two heavy nuclei part of their kinetic energy in the center of mass frame transforms into intense heat before the system reaches the thermal equilibrium, leading to a strongly coupled plasma. When the collision occurs the initial state represents a far-from-equilibrium system. A proposal to model such a system has been the so-called holographic thermalization, where it is assumed that there is a sudden injection of energy to the system which in the thermal quantum field theory is interpreted as a thermal quench. 
The time evolution of the thermalizing system is a very complicated process to model due to the large number of degrees of freedom involved as well as its non-perturbative character.

We consider a model of dynamical holographic thermalization [2-17] which has been recently used to study thermalization time scale of strongly coupled plasmas. Although most of these applications have been done by using effective holographic dual models, in the present work we focus on an specific string theory dual model in order to investigate quantum corrections to the holographic thermalization time scale of a particular strongly coupled SYM plasma whose string dual description is very well known. We can schematically describe this thermalization model starting from an initial vacuum gravity solution given by an anti-de Sitter spacetime (AdS), which represents the holographic dual description of a certain quantum field theory at zero temperature. The final state is defined by an asymptotically anti-de Sitter Schwarzschild black hole (AdS-BH), with Hawking temperature $T_{H}$, which is the gravity dual model corresponding to the quantum field theory at finite equilibrium temperature $T$. Furthermore, it is assumed that $T=T_{H}$. Interestingly, there is a solution of the Einstein equations which interpolates between these gravity solutions, and it can be represented by a thin shell collapsing from the AdS-boundary. The shell separates the space into two regions: the inner one is an AdS and the outer one is an AdS-BH. As we shall explain, extended probes in the bulk can be used in order to measure the thermalization time scale of the boundary theory plasma.

The general picture which emerges from the gauge/gravity duality when studying the dynamical evolution of a collapsing thin shell shows that UV modes thermalize faster than IR ones. This statement is based upon the fact that extended geometric probes in the bulk, such as space-like geodesic curves, minimal area surfaces and minimal volume hyper-surfaces, thermalize faster when the boundary field theory separation between two operators (which are dual to these bulk geometric probes) becomes shorter. Thus, the claim is that the faster thermalization of short distance two-point functions of quantum field theory operators of the boundary field theory is related to faster thermalization of UV modes, in comparison with IR modes which are associated with two-point functions of operators with larger separation between them.

The referred behavior has been found for very different kinds of degrees of freedom composing the shell [17] in effective five-dimensional holographic dual models, and from the gravity point of view it follows as a consequence of the construction of the geometric probes. In particular, this also occurs in the planar limit of the $\mathrm{SU}(N) \mathcal{N}=4 \mathrm{SYM}$ plasma at the strong 't Hooft coupling $(\lambda)$ limit. This result is obtained from its dual supergravity description, i.e. with no string theory corrections. Thus, a natural question which arises is whether string theory quantum corrections modify or not that statement about UV/IR modes thermalization, and if they do, it is crucial to know how are such corrections. In this paper we address this question by considering the leading string theory corrections to type IIB supergravity. Very interestingly, we find the emergence of an energy scale which separates the thermalization time scales of IR and UV modes. Thus, by decreasing $\lambda$ the thermalization time scale for IR modes slightly increases in comparison with its thermalization time scale at the $\lambda \rightarrow \infty$ limit, when final states are compared at fixed temperature or fixed energy, i.e. they thermalize a bit slower than in the strong coupling limit. On 
the other hand, the alluded corrections induce an opposite behavior on the thermalization time for UV modes, i.e. they thermalize slightly faster compared with the strong coupling limit. We shall discuss about these two distinctive effects in the last section of the paper. We also consider the effect of string-loop corrections which lead to $1 / N$ corrections in the dual SYM theory, and show that their effects go in the same direction as for the mentioned higher curvature corrections.

In particular, we study the dynamical evolution of a thin shell composed by massless type IIB supergravity degrees of freedom, collapsing within the asymptotically AdS spaces described before. This is aimed at investigating holographic thermalization of $\operatorname{SU}(N) \mathcal{N}=$ 4 SYM theory plasma at strong yet finite coupling. On the boundary quantum field theory this corresponds to considering a certain thermal quench. As we have already commented, we assume it corresponds to a sudden injection of energy due to a heavy-ion collision. Essentially, this energy is a fraction of the center of mass kinetic energy of the two colliding heavy ions which is transformed into intense heat during the plasma thermalization. In a certain way this energy enters the definition of the parameter $M$ introduced in the thermal quench. We study the thermalization time scale by calculating renormalized space-like geodesic lengths, rectangular and circular minimal area surfaces, and three-dimensional minimal volume hyper-surfaces, as extended probes of thermalization, which are claimed to be related to two-point functions, rectangular and circular Wilson loops, and entanglement entropy, respectively. Thus, on the quantum field theory side we consider different non-local observables to probe thermalization of this strongly coupled system.

We consider three scenarios described in terms of the parameters of the model, which are the equilibrium temperature $T$ and the energy parameter $M$ of the quench, which are related each other by eq. (2.17). One situation is when we fix the horizon radius $z_{h}$ or equivalently the parameter $M$ (see eq. (2.2)), independently of the quantum corrections, this makes the equilibrium temperature to be dependent of the 't Hooft coupling. Secondly, we consider the case when the equilibrium temperature is kept fixed, while the horizon radius becomes a function of the 't Hooft coupling. These two situations show the same behavior for the thermalization, as explained before. Then, we discuss what happens when we vary the parameter $M$ of the thermal quench in an arbitrary way, which we associate with the fraction of the center of mass kinetic energy of the two colliding heavy ions which transforms into heat and initiates the thermalization process. We discuss in detail these three cases in section 4 .

Notice that recently higher curvature corrections to the $\mathrm{SU}(N) \mathcal{N}=4 \mathrm{SYM}$ plasma thermalization using a quasi-static approximation have been discussed in $[18,19]$, obtaining a different behavior for the UV and IR modes compared with our results. However, one has to be cautious making that comparison since in these references the $\mathrm{SU}(N) \mathcal{N}=4 \mathrm{SYM}$ theory is coupled to an external electromagnetic field, which induces large $\mathcal{O}\left(\alpha^{\prime 3}\right)$ corrections for certain observables. In section 5 we shall discuss more about this in comparison with our findings.

The paper is organized as follows. In section 2 we describe the leading string theory corrections to the type IIB supergravity action, including string-loop corrections. In section 3 we consider the quantum corrections to the holographic thermalization process. Results and discussions are introduced in section 4 . Section 5 is devoted to the conclusions. 


\section{Leading type IIB string theory corrections}

The ten-dimensional $\mathrm{AdS}_{5}-\mathrm{BH} \times S^{5}$ metric is given by

$$
d s^{2}=\frac{R^{2}}{z^{2}}\left[-f(z) d t^{2}+d \vec{x}^{2}+\frac{1}{f(z)} d z^{2}\right]+R^{2} d \Omega_{5}^{2}
$$

This is in fact an exact solution of type IIB supergravity, which turns out to be the metric of the background of the holographic dual model corresponding to the planar limit of the $\mathrm{SU}(N) \mathcal{N}=4 \mathrm{SYM}$ theory at finite temperature, $T$, in the strong coupling limit. In the above expression we define

$$
f(z)=1-\frac{z^{4}}{z_{h}^{4}}=1-2 M z^{4}
$$

while the radius of the $\mathrm{AdS}_{5}$ and the five-sphere is $R$. At $z=0$ we have the boundary of the $\mathrm{AdS}_{5}$ space, while the black hole horizon is set at $z=z_{h}$.

Now, let us consider the leading type IIB string theory corrections to the supergravity action $S_{I I B}^{S U G R A}$. The corrections are described by the term $S_{\mathcal{R}^{4}}^{3}$. Therefore, the total action at $\mathcal{O}\left(\alpha^{\prime 3}\right)$ can be written as follows

$$
S_{I I B}=S_{I I B}^{S U G R A}+S_{\mathcal{R}^{4}}^{3}
$$

Since the square root of the 't Hooft coupling is related to the inverse of $\alpha$ ', at the strong 't Hooft coupling limit $(N \gg \lambda \gg 1)$ the holographic dual model is derived from type IIB supergravity, i.e. for $\alpha^{\prime} \rightarrow 0$. In particular, $S_{I I B}^{S U G R A}$ is composed by the Einstein-Hilbert action coupled to the dilaton and the Ramond-Ramond five-form field strength $F_{5}$. In the Einstein frame it reads

$$
S_{I I B}^{S U G R A}=\frac{1}{2 \kappa_{10}^{2}} \int d^{10} x \sqrt{-G}\left[R_{10}-\frac{1}{2}(\partial \phi)^{2}-\frac{1}{4.5 !}\left(F_{5}\right)^{2}\right] .
$$

By considering D3-branes in type IIB string theory, in reference [20] the contributions from higher curvature terms at $\mathcal{O}\left(\alpha^{\prime 3}\right)$, as well as perturbative $1 / N$ corrections and instanton corrections were computed. The idea is to look at a supersymmetric completion of the $C^{4}$ term, where $C$ is the ten-dimensional Weyl tensor. Thus

$$
S_{\mathcal{R}^{4}}^{3}=\frac{\alpha^{\prime 3} g_{s}^{3 / 2}}{32 \pi G} \int d^{10} x \int d^{16} \theta \sqrt{-g} f^{(0,0)}(\tau, \bar{\tau})\left[\left(\theta \Gamma^{m n p} \theta\right)\left(\theta \Gamma^{q r s} \theta\right) \mathcal{R}_{m n p q r s}\right]^{4}+c . c .,
$$

where $\tau$ is the complex scalar field written as $\tau_{1}+i \tau_{2} \equiv a+i e^{-\phi}$, where $a$ is the axion, $\phi$ is the dilaton and $e^{\phi}=g_{s}$ is the string coupling. Recall that in terms of the gauge/string duality $g_{s} \equiv 1 / N$. In the above expression $f^{(0,0)}(\tau, \bar{\tau})$ is the modular form. Using the Weyl tensor one can define the tensor $\mathcal{R}[20-23]$

$$
\mathcal{R}_{\text {mnpqrs }}=\frac{1}{8} g_{p s} C_{m n q r}+\frac{i}{48} D_{m} F_{n p q r s}^{+}+\frac{1}{384} F_{m n p k l}^{+} F_{q r s}^{+k l},
$$

where

$$
F^{+}=(1+*) F_{5} / 2 .
$$


Notice that the action (2.5) has been obtained using the fact that the physical field content of type IIB supergravity can be arranged in a scalar superfield $\Phi(x, \theta)$, where $\theta_{a}$, with $a=1, \cdots, 16$, is a complex Weyl spinor of $\mathrm{SO}(1,9)$. The matrices $\Gamma$ have been defined as usual [22]. The modular form is given by the following expression [24]

$$
f^{(0,0)}(\tau, \bar{\tau})=2 \zeta(3) \tau_{2}^{3 / 2}+\frac{2 \pi^{2}}{3} \tau_{2}^{-1 / 2}+8 \pi \tau_{2}^{1 / 2} \sum_{m \neq 0, n \geq 0} \frac{|m|}{|n|} e^{2 \pi i|m n| \tau_{1}} K_{1}\left(2 \pi|m n| \tau_{2}\right),
$$

where $K_{1}$ is the modified Bessel function of second kind which comes from the non-perturbative D-instantons contributions. The zeta function $\zeta(3)$ is the coefficient of the first perturbative correction in the Eisenstein series of the modular form. Also note that in the background we consider with $N$ coincident parallel D3 branes there are some simplifications: the axion vanishes, thus $\tau_{1}=0$, while $\tau_{2}=g_{s}^{-1}$. Thus, when the string coupling is small the modular form reduces to

$$
f^{(0,0)}(\tau, \bar{\tau})=2(4 \pi N)^{3 / 2}\left(\frac{\zeta(3)}{\lambda^{3 / 2}}+\frac{\lambda^{1 / 2}}{48 N^{2}}+\frac{e^{-8 \pi^{2} N / \lambda}}{2 \pi^{1 / 2} N^{3 / 2}}\right) .
$$

It has been also shown that the D3-brane solution in supergravity is not renormalized by higher derivative terms [20]. Previously, Banks and Green had shown that $A d S_{5} \times S^{5}$ is a solution to all orders in $\alpha^{\prime}$ [25]. This is not the case for the AdS-BH we consider, whose metric does receive corrections at order $\alpha^{\prime 3}$ as we shall see shortly.

At this point, let us also consider the large $N$ limit of the dual $\mathrm{SU}(N) \mathcal{N}=4 \mathrm{SYM}$ theory. The finite leading 't Hooft coupling corrections in its string theory dual description are accounted for by the following action [23]

$$
S_{I I B}^{\alpha^{\prime}}=\frac{R^{6}}{2 \kappa_{10}^{2}} \int d^{10} x \sqrt{-G}\left[\gamma e^{-\frac{3}{2} \phi}\left(C^{4}+C^{3} \mathcal{T}+C^{2} \mathcal{T}^{2}+C \mathcal{T}^{3}+\mathcal{T}^{4}\right)\right],
$$

which was obtained from the action (2.5) in the planar limit of the $\mathrm{SU}(N) \mathcal{N}=4 \mathrm{SYM}$ theory. Notice that we have written this $\alpha^{\prime 3}$-corrected action (2.10) in the Einstein frame. Here $\gamma \equiv \frac{1}{8} \zeta(3)\left(\alpha^{\prime} / R^{2}\right)^{3}$, where $R^{4}=4 \pi g_{s} N \alpha^{\prime 2}$. Since $\lambda=g_{Y M}^{2} N \equiv 4 \pi g_{s} N$, therefore $\gamma=\frac{1}{8} \zeta(3) \frac{1}{\lambda^{3 / 2}}$. This action was computed in [22], using the methods of [26].

The $C^{4}$ term is a dimension-eight operator, given by

$$
C^{4}=C^{h m n k} C_{p m n q} C_{h}^{r s p} C_{r s k}^{q}+\frac{1}{2} C^{h k m n} C_{p q m n} C_{h}^{r s p} C_{r s k}^{q},
$$

where $C^{q}{ }_{r s k}$ is the Weyl tensor. The tensor $\mathcal{T}$ is much more involved and it is defined by

$$
\mathcal{T}_{\text {abcdef }}=i \nabla_{a} F_{b c d e f}^{+}+\frac{1}{16}\left(F_{a b c m n}^{+} F_{d e f}^{+m n}-3 F_{a b f m n}^{+} F_{d e c}^{+m n}\right)
$$

where the indices $[a, b, c]$ and $[d, e, f]$ are antisymmetrized in each squared brackets, and symmetrized with respect to interchange of $a b c \leftrightarrow d e f[22]$.

Importantly, at finite temperature the metric only has corrections coming from the $C^{4}$ term. This is so because the tensor $\mathcal{T}$ vanishes on the supergravity solution with no string theory corrections [23]. The solution to the Einstein equations derived from the 
supergravity action (2.4) is an $\mathrm{AdS}_{5}-\mathrm{BH} \times S^{5}$. There are $N$ units of flux of $F_{5}$ through the sphere. Recall that $N$ is the rank of the gauge group in the field theory, and on the other hand, it corresponds to the number of parallel D3-branes whose back-reaction deforms the space-time leading to the above metric in the near horizon limit.

In conclusion, the only part of the $\mathcal{O}\left(\alpha^{\prime 3}\right)$-action which affects the metric is the $C^{4}$ term. This induces the following corrected metric obtained by [27-29]

$$
d s^{2}=\frac{R^{2}}{z^{2}}\left[-f(z) K^{2}(z) d t^{2}+d \vec{x}^{2}+f^{-1}(z) P^{2}(z) d z^{2}\right]+R^{2} L^{2}(z) d \Omega_{5}^{2},
$$

using same notation as in eq. (2.1). $K(z), P(z)$ and $L(z)$ are given by the following expressions:

$$
K(z)=e^{\gamma[a(z)+4 b(z)]}, \quad P(z)=e^{\gamma b(z)}, \quad L(z)=e^{\gamma c(z)},
$$

where

$$
\begin{aligned}
& a(z)=-\frac{1625}{8}\left(\frac{z}{z_{h}}\right)^{4}-175\left(\frac{z}{z_{h}}\right)^{8}+\frac{10005}{16}\left(\frac{z}{z_{h}}\right)^{12}, \\
& b(z)=\frac{325}{8}\left(\frac{z}{z_{h}}\right)^{4}+\frac{1075}{32}\left(\frac{z}{z_{h}}\right)^{8}-\frac{4835}{32}\left(\frac{z}{z_{h}}\right)^{12}, \\
& c(z)=\frac{15}{32}\left(1+\left(\frac{z}{z_{h}}\right)^{4}\right)\left(\frac{z}{z_{h}}\right)^{8} .
\end{aligned}
$$

In addition, after the leading type IIB string theory corrections are taken into account, the dilaton field becomes $\phi=\phi_{0}+\gamma \phi_{1}+\mathcal{O}\left(\gamma^{2}\right)$, where

$$
\begin{aligned}
\phi_{0} & =-\log \left(g_{s}\right), \\
\phi_{1}(z) & =-\frac{45}{8}\left(\frac{z^{4}}{z_{h}^{4}}+\frac{1}{2} \frac{z^{8}}{z_{h}^{8}}+\frac{1}{3} \frac{z^{12}}{z_{h}^{12}}\right) .
\end{aligned}
$$

The temperature of the boundary field theory, which is assumed to be equal to the Hawking temperature of the $\mathrm{AdS}_{5}$-Schwarzschild black hole, is now corrected as [28]

$$
T=\frac{(2 M)^{\frac{1}{4}}}{\pi R^{2}}\left(1+\frac{265}{16} \gamma\right)
$$

Having obtained the corrected metric, dilaton, and temperature, equations (2.13), (2.16) and (2.17), we now focus on the leading quantum corrections to the holographic thermalization process.

\section{Quantum corrections to holographic thermalization}

\subsection{A collapsing thin shell in Anti de Sitter space}

The AdS-Vaidya solution has been widely employed to account for the evolution towards the final equilibrium state of strongly coupled SYM plasmas [2-17]. This solution describes the geometry of a pressureless thin shell composed by massless particles collapsing 
in an AdS spacetime, ${ }^{1}$ leading to the formation of a black hole with negative cosmological constant at asymptotic times.

Since the shell is composed by coherent massless degrees of freedom, it moves at the speed of light. Thus, it is set at a constant position in the Eddington-Filkenstein-like coordinate $v_{0}$, defined by

$$
d v_{0}=d t-f^{-1}(z) d z
$$

The AdS part of the metric describing this geometry, in the thin-shell limit, takes the following form

$$
d s^{2}= \begin{cases}\frac{R^{2}}{z^{2}}\left[-d v_{0}^{2}-2 d v_{0} d z+d \vec{x}^{2}\right], & v_{0}<0 \\ \frac{R^{2}}{z^{2}}\left[-f(z) d v_{0}^{2}-2 d v_{0} d z+d \vec{x}^{2}\right], & v_{0}>0\end{cases}
$$

Once the corrections to the pure type IIB supergravity solution are considered, the null dust and zero width hypotheses lead to a geometry with the metric given by

$$
d s^{2}= \begin{cases}\frac{R^{2}}{z^{2}}\left[-d v^{2}-2 d v d z+d \vec{x}^{2}\right], & v<0, \\ \frac{R^{2}}{z^{2}}\left[-f(z) K^{2}(z) d v^{2}-2 P(z) K(z) d v d z+d \vec{x}^{2}\right], & v>0,\end{cases}
$$

where $v$ is now defined by

$$
d v=d t-\frac{e^{-\gamma[a(z)+3 b(z)]}}{f(z)} d z
$$

\subsection{Probing thermalization: non-local observables}

In order to probe the thermalization one must consider the evolution of different nonlocal observables. In the AdS/CFT correspondence such boundary quantum field theory observables are typically identified with geometric objects in the bulk, e.g. the Wightman two-point function of scalar field operators is determined by the sum of curve lengths ending at the location of the operators. In the limit of highest conformal weights, the saddle-point approximation reduces the sum just to the contribution of the shortest length, i.e. the geodesic length [31]. Similarly, in the classical limit, the Wilson loops are determined by minimal area surfaces ending on the closed Wilson path [32], and the entanglement entropy of a given volume in the boundary theory is set by the minimal bulk hyper-surface ending in such a region $[33,34]$.

Equation (3.4) determines the position of the shell as a function of the boundary time, $t$. Without lost of generality we can set $t=0$ as the moment the shell leaves the boundary. Thus, a boundary observer feels that for any $t>0$ there is a spatial scale $\tilde{l}$ at the boundary such that the extended geometric objects in the bulk (like geodesic curves and hyper-surfaces) with ends at the boundary whose separation is smaller than $\tilde{l}$ are completely

\footnotetext{
${ }^{1}$ It is worth mentioning that AdS-Vaidya metric (3.2) is not an exact solution of type IIB supergravity on $A d S_{5} \times S^{5}$. Nevertheless, it was shown [30] that this solution appears as a good approximation of certain field configurations.
} 
embedded in the AdS-BH region of the bulk. Therefore, the observable associated quantities of the boundary theory exactly match their corresponding measures of the plasma in thermal equilibrium.

On the other hand, extended objects probing larger scales than $\tilde{l}$ on the boundary have to wait more time before they agree with their corresponding thermally equilibrated values. This simple geometric picture gives us a very simple (gravity) intuition of why, contrary to what happens in perturbative theories, UV modes thermalize before than the IR ones do.

Once quantum corrections are included non-local observables may no longer be given by the geometric quantities described above. ${ }^{2}$ Nevertheless, there is evidence supporting the statement that the new definition for these non-local observables is not given in terms of the length of the geodesic or the area/volume of the minimal hypersurface, but in terms of a different functional over the same geodesic or minimal surfaces. Thus, provided that we exclusively focus on thermalization times, and not on the precise value of the nonlocal observables, we still have to consider the same kind of objects considered before the introduction of $\alpha^{\prime}$ corrections.

For instance, let us consider a scalar field coupled to gravity through a standard interaction term

$$
S_{\text {Int }}(g, \phi)=\frac{1}{2} \int d^{D} x \sqrt{-g}\left(\partial_{\mu} \phi \partial_{\nu} \phi+m^{2} \phi^{2}\right) .
$$

Then, following [35] with the new effective supergravity action, the two-point function is now given by the old one with the replacement of the AdS-Schwarzschild metric by the corrected one (2.13). This is up to an overall factor containing the Weyl tensor (2.10), which plays no role in the variation with respect to the boundary values of the scalar fields. Thus, following [31], the corrected two-point function in the large conformal weight limit is, up to an overall constant, given by the exponential of the geodesic length in the space whose metric is defined by equation (2.13).

Concerning the entanglement entropy, at the present it is not known a general definition for it if one considers higher derivative gravity. Some progress in this direction was achieved for Gauss-Bonnet and Lovelock gravities. In this situation, it was shown in [36], that Wald's formula used by [33] does not satisfy the subadditivity condition when higher derivative terms are included in the Einstein-Hilbert action. They found a natural generalization which replaces the projection of the curvature tensor by the intrinsic curvature tensor of the surface in the Wald's formula. This new definition was used for the GaussBonnet gravity, as well as in the next order in derivatives (see (C.21) and (C.28) of [36]) of Lovelock gravity. These definitions reproduce the expressions derived in [37] for an arbitrary higher curvature theory, and although they do not agree with the Ryu-Takayanagi (RT) prescription, these new functionals are computed over the same minimal surfaces used in the RT prescription (see (C.14) of [36]).

Recent studies [38] have found that entanglement entropy at $T=0$ does not receive corrections when leading higher derivative terms from type IIB string theory are considered, studying leading 't Hooft coupling corrections to $\mathcal{N}=4$ SYM theory. Thus, although

\footnotetext{
${ }^{2}$ We thank Robert Myers for some comments about this point.
} 
the Einstein-Hilbert action is supplemented with the Weyl term (2.10), the entanglement entropy is computed in terms of the same minimal surface used in the RT prescription.

Now, let us consider Wilson loops. It is worth nothing that these observables are not computed in terms of the supergravity action. Instead, one directly uses the string theory action for curved backgrounds [32]. Then, $\alpha^{\prime}$ corrections are analyzed in a different way. They correct the background, but they also demand to consider quadratic fluctuations around the classical solution. Although the later give the leading corrections to the Wilson loop (order $\mathcal{O}\left(\alpha^{\prime}\right)$ with respect to the classical solution), they are seen as wrinkles of the classical surface, and they are not expected to be relevant for the discussion of thermalization times. A quantitative estimate of this claim is out of the scope of the present work, nevertheless, our results displayed on the figures in the following section strongly support this statement. This is so, because the conclusions for thermalization times obtained by using different observables are nearly the same.

Wightman two-point function. Equal time two-point correlation functions of boundary quantum field theory operators are related to space-like geodesics. In the AdS-BH region of the bulk these are the ones that minimize the functional length

$$
\mathcal{L}=R \int_{-\frac{\ell}{2}}^{\frac{\ell}{2}} d x \frac{\sqrt{1-2 e^{\gamma(a(z)+5 b(z))} v^{\prime}(x) z^{\prime}(x)-e^{\gamma(2 a(z)+8 b(z))} f(z) v^{\prime}(x)^{2}}}{z(x)}
$$

where $x$ is one of the $x^{i}$-coordinates on the boundary and the rest have been chosen to be fixed along the curve. Since there is no explicit dependence on the $x$ variable, the associated "Hamiltonian" is a constant of motion. It leads to the equation

$$
1-2 e^{\gamma(a(z)+5 b(z))} v^{\prime}(x) z^{\prime}(x)-e^{\gamma(2 a(z)+8 b(z))} f(z) v^{\prime}(x)^{2}=\left(\frac{z^{*}}{z}\right)^{2},
$$

where we have used the fact that $v(x)$ and $z(x)$ have a maximum at $x=0$, and we have introduced $z^{*}=z(0)=z_{\max }$.

On the other hand, the time independence of the metric in the AdS-BH region requires $t^{\prime}(x)=0$ as solution to the boundary conditions $t\left(\frac{\ell}{2}\right)=t\left(-\frac{\ell}{2}\right)$. It leads to

$$
v^{\prime}(x)=-\frac{e^{-\gamma[a(z)+3 b(z)]}}{f(z)} z^{\prime}(x),
$$

which together with equation (3.7) can be used to express $z^{\prime}(x)$ as a function of $z$. Furthermore, one can use it to obtain the following expression for the separation of the geodesic ends

$$
\ell=2 \int_{0}^{z^{*}} \frac{d z}{\sqrt{e^{-2 \gamma b(z)} f(z)\left[\left(\frac{z^{*}}{z}\right)^{2}-1\right]}} .
$$

Notice that this has been derived within the Einstein frame. 
Rectangular Wilson loops. Wilson loops are computed in terms of the Polyakov action for curved spaces [32]. Since we are working within the Einstein frame, the dilaton appears in two different ways in the action, namely: in a conformal factor connecting the string metric with the Einstein metric, and also multiplying the world-sheet scalar curvature. The later is order $\alpha^{\prime}$ with respect to the metric contribution, therefore, the leading $\phi_{0}$ term gives rise to the string coupling constant while $\phi_{1}$ leads to a sub-leading contribution with respect to the metric corrections, so they are neglected here. Concerning the conformal factor, it is actually a function of $\phi-\phi_{0}$, so it introduces only $\phi_{1}(z)$ in the action.

Then, the minimal area surface related to space-like rectangular Wilson loop with edges of length $\ell$ and $L$, satisfying $L \gg \ell$, is determined by the functional

$$
\mathcal{A}=R^{2} L \int_{-\frac{\ell}{2}}^{\frac{\ell}{2}} d x e^{\frac{1}{2} \gamma \phi_{1}(z)} \frac{\sqrt{1-2 e^{\gamma(a(z)+5 b(z))} v^{\prime}(x) z^{\prime}(x)-e^{\gamma(2 a(z)+8 b(z))} f(z) v^{\prime}(x)^{2}}}{z(x)^{2}} .
$$

Following similar steps as for the geodesic length case one finds

$$
\ell=2 \int_{0}^{z^{*}} \frac{d z}{\sqrt{e^{-2 \gamma b(z)} f(z)\left[\left(\frac{z^{*}}{z}\right)^{4} e^{\gamma\left(\phi_{1}(z)-\phi_{1}\left(z^{*}\right)\right)}-1\right]}} .
$$

Circular Wilson loops. The minimal area surface associated with a space-like circular Wilson loop with radius $\mathcal{R}$ is given by

$$
\mathcal{A}=2 \pi R^{2} \int_{0}^{\mathcal{R}} d \rho \frac{\rho}{z(\rho)^{2}} e^{\frac{1}{2} \gamma \phi_{1}(z)} \sqrt{1-2 e^{\gamma(a(z)+5 b(z))} v^{\prime}(\rho) z^{\prime}(\rho)-e^{\gamma(2 a(z)+8 b(z))} f(z) v^{\prime}(\rho)^{2}} .
$$

Unfortunately, there is no constant of motion associated to $\rho$ in this case. Nevertheless, we can use the equation coming from the condition $t^{\prime}(\rho)=0$ to replace $v^{\prime}(\rho)$ as a function of $\left(z(\rho), z^{\prime}(\rho)\right)$ in equation (3.12), leading to the following equation of motion

$$
\begin{aligned}
& -\frac{1}{2} \gamma \partial_{z} \phi_{1} \rho z(\rho) z^{\prime}(\rho)\left(1-z(\rho)^{4}\right)\left(1-z(\rho)^{4}+e^{2 \gamma b} z^{\prime}(\rho)^{2}\right) \\
& +\left(1-z(\rho)^{4}+e^{2 \gamma b(z)} z^{\prime}(\rho)\right)\left(2 \rho\left(1-z(\rho)^{4}\right)+e^{2 \gamma b(z)} z(\rho) z^{\prime}(\rho)\right)+2 \rho e^{2 \gamma b(z)} z(\rho)^{4} z^{\prime}(\rho)^{2} \\
& +\frac{1}{2}\left(1-z(\rho)^{4}\right) \rho \partial_{z}\left(e^{2 \gamma b(z)}\right) z(\rho) z^{\prime}(\rho)^{2}+\left(1-z(\rho)^{4}\right) \rho e^{2 \gamma b(z)} z(\rho) z^{\prime \prime}(\rho)=0
\end{aligned}
$$

No analytical solution is known for this non-linear differential equation. Thus, expressions with $\mathcal{R}$ as a function of $z^{*}$ are only numerically available.

Entanglement entropy. Now, we shall focus on the entanglement entropy of spherical regions at the boundary theory. The minimal volume of the bulk hyper-surface ending on it is specified by

$$
\mathcal{V}=4 \pi R^{3} \int_{0}^{\mathcal{R}} d \rho \frac{\rho^{2}}{z(\rho)^{3}} \sqrt{1-2 e^{\gamma(a(z)+5 b(z))} v^{\prime}(\rho) z^{\prime}(\rho)-e^{\gamma(2 a(z)+8 b(z))} f(z) v^{\prime}(\rho)^{2}} .
$$

After following similar steps as for the circular Wilson loop case one obtains the following differential equation

$$
\left(1-z(\rho)^{4}+e^{2 \gamma b(z)} z^{\prime}(\rho)\right)\left(3 \rho\left(1-z(\rho)^{4}\right)+2 e^{2 \gamma b(z)} z(\rho) z^{\prime}(\rho)\right)+2 \rho e^{2 \gamma b(z)} z(\rho)^{4} z^{\prime 2}(\rho)
$$




$$
+\frac{1}{2}\left(1-z(\rho)^{4}\right) \rho \partial_{z}\left(e^{2 \gamma b(z)}\right) z(\rho) z^{\prime 2}(\rho)+\left(1-z(\rho)^{4}\right) \rho e^{2 \gamma b(z)} z(\rho) z^{\prime \prime}(\rho)=0 .
$$

Again, $\mathcal{R}$ can be expressed as a function of $z^{*}$ only numerically.

In the classical limit, where all these non-local observables are well approximated as functions of these geometric objects, the thermalization at a given scale is determined by the position of the shell at that moment. If all non-local observables testing a particular scale have their associated $z^{*}$ smaller than $z(t)_{\text {shell }}$, we consider that this scale has thermalized. In the next section we shall consider the thermalization time as a function of the boundary scale, e.g. the separation between two operator insertions at the boundary theory, between Wilson lines, or entangled region, for the different non-local observables discussed here once quantum corrections are taken into account.

\section{Results and discussion}

It is worth noting that the shell moves slower near the boundary as $\lambda$ decreases from the strong coupling limit. However, $z^{*}(\ell)$ and $z^{*}(\mathcal{R})$ decrease in such a way that the net result renders a faster thermalization. Also notice that the opposite behavior is observed near the black hole horizon.

The results for the thermalization times are displayed in figure 1. The sequence of curves: blue, green, brown and red correspond to $\lambda \rightarrow \infty, 150,70,30$, respectively and $z_{h}=1$. All non-local probes used to study thermalization show that there exist a critical length, which at least for the range studied $\left(0.5<z_{h}<5\right)$ is located about $(1.7-1.8) \times z_{h}$, for two-point functions, circular Wilson loops and entanglement entropy, and about $0.95 \times z_{h}$ for rectangular Wilson loops. Shorter distances than the critical scale measure a reduction in the thermalization time, while larger distances perceive a delay. Figure 2 shows only the $\alpha^{\prime 3}$ corrections for case of the geodesics in order to display more clearly the crossover point. Of course, as the UV modes thermalize near instantaneously, their $\alpha^{\prime}$ corrections are hardly appreciable. Another distinction between rectangular Wilson loops and the rest of the observables is a delay in reaching the equilibrium state (see figure 1.b). This effect is likely to be a consequence of the fact that rectangular Wilson loops are not completely contained in a region of size $\ell$, because of the presence of the scale $L$, which is a second large scale for this particular observable. This is what makes a difference with respect to the other non-local observables discusses in this work. Notice that although the geodesic lengths and minimal surfaces depend on $R$, the thermalization times do not depend on $R$, so $z_{h}$ is the unique relevant scale to this discussion. ${ }^{3}$

We have considered corrections to thermalization time for systems with the same amount of energy parameter $M$ at different strong couplings, $\lambda$. Another interesting scenario is to compare thermalization time for systems evolving to a given thermal equilibrium temperature. By doing so, instead of a fixed $z_{h}$, it requires (see eq. (2.17))

$$
z_{h}=z_{h}^{(0)} \rightarrow z_{h}^{(0)}\left(1+\frac{265}{16} \gamma\right)
$$

\footnotetext{
${ }^{3}$ It is worth mentioning that the situation is different if one changes the degrees of freedom of the shell. As discussed in [17] and displayed in figure 2 of loc.cit., when the shell is not composed by a massless pressureless fluid, the velocity of the shell depends on the radius of AdS space.
} 


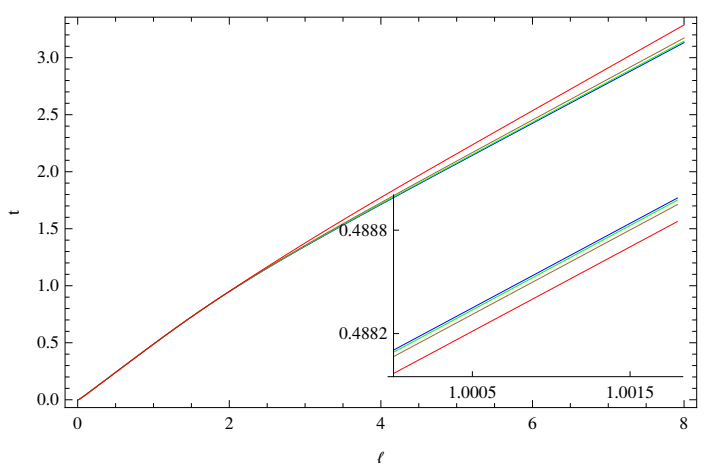

(a) Two-point function.

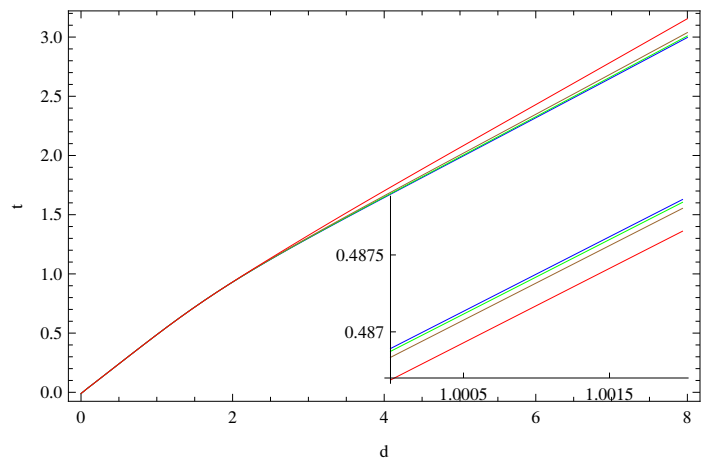

(c) Circular Wilson loop.

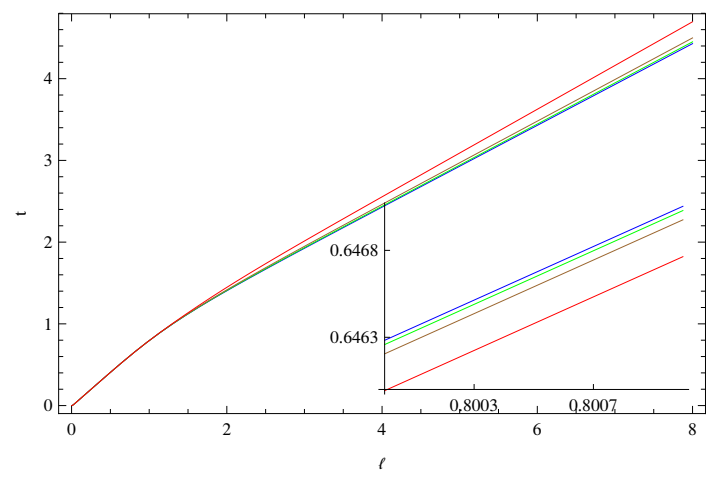

(b) Rectangular Wilson loop.

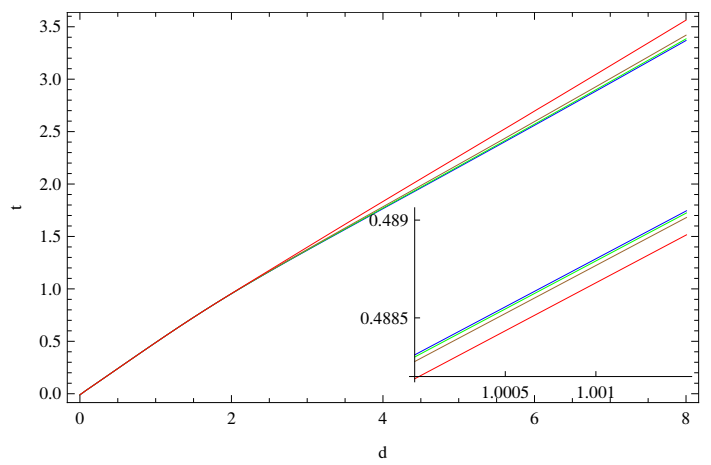

(d) Entanglement entropy.

Figure 1. Figures show thermalization times as functions of spatial resolution on the boundary theory. In figures (a) and (b), $\ell$ denotes geodesic separation and separation for the rectangular Wilson loop, respectively. In figures (c) and (d), $d=2 \mathcal{R}$ denote the diameter of the circular Wilson loop and of the sphere associated with the entanglement entropy. Blue curves describe the thermalization pattern without $\alpha^{\prime}$ corrections $(\lambda \rightarrow \infty)$, the green ones correspond to $\lambda=150$, brown to $\lambda=70$ and the red ones to $\lambda=30$.

In this situation, the thermalization is still very similar to the previous case, the only difference is that the crossover point is shifted to the IR and is located at about $(1.9-2.1) \times z_{h}$.

Although we are analyzing an $\mathcal{N}=4 \mathrm{SYM}$ plasma, one is tempted to compare the thermalization patterns with more realistic scenarios like strongly coupled QGP of RHIC and LHC experiments. In these situations two heavy ions collide at relativistic velocities and after that thousand of particles are created and a fraction of the kinetic energy of the original nuclei is converted into heat leading to a finite temperature strongly coupled plasma.

It means that in order to analyze more rigorously the pattern of thermalization times with large yet finite coupling, one should be able to model the amount of kinetic energy which is converted into heat during the collision, when the coupling decreases from infinity to a given finite value. This information is an input for our approach which enters the mass of the collapsing shell. The knowledge of this information in the case of QGP requires that one should be able to model the whole process: collision, deconfinement and 


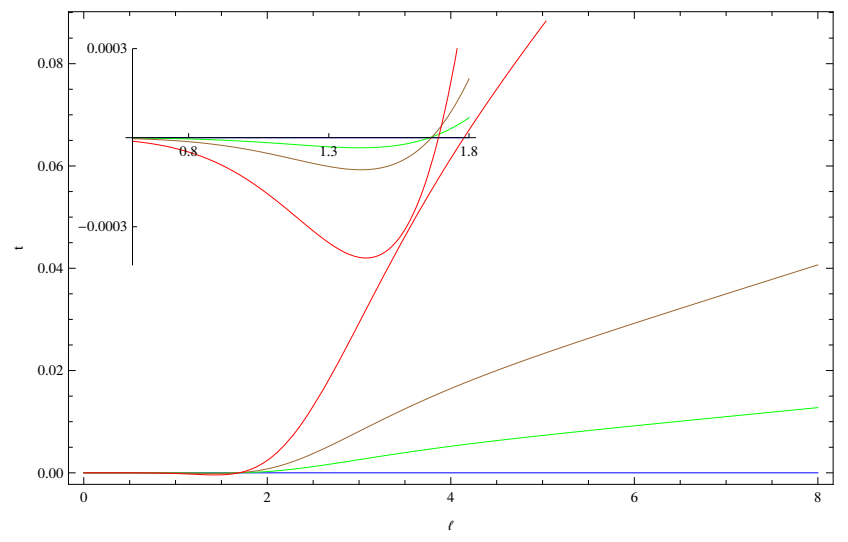

Figure 2. This plot displays the $\alpha^{\prime}$ corrections to the thermalization time as a function of the scale resolution in the case of two-point functions. The identification among colors and couplings is as in figure 1 .

thermalization. However, since $\mathcal{N}=4 \mathrm{SYM}$ is not a confining quantum field theory, it is not expected that this information could be inferred from our analysis.

Numerical analysis shows that if the rate of kinetic energy transferred increases, the critical scale shifts towards the IR (as for instance in the fixed-temperature scenario). On the other hand, if it decreases ( $z_{h}$ increases) there exist a bound $z_{h} \rightarrow z_{h}(1+\Lambda \gamma), \Lambda \lesssim 20$, where there is still a critical length, and above this limit any scale experiences a delay in reaching the thermal equilibrium.

We have not displayed here the results with $1 / N$ corrections. Nevertheless, it is easy to see that there is no qualitative difference. In fact, there is only a hardly appreciable difference with respect to the case with $\alpha^{\prime}$ corrections. The effective value for $\gamma$, when both $\alpha^{\prime}$ and $1 / N$ corrections are considered, is given by (see equation (2.9))

$$
\gamma=\frac{1}{8} \zeta(3) \frac{1}{\lambda^{\frac{3}{2}}} \rightarrow \frac{1}{8} \frac{1}{\lambda^{\frac{3}{2}}}\left(\zeta(3)+\frac{1}{48}\left(\frac{\lambda}{N}\right)^{2}+\frac{1}{2 \pi^{1 / 2}}\left(\frac{\lambda}{N}\right)^{\frac{3}{2}} e^{-8 \pi^{2} \frac{N}{\lambda}}\right) .
$$

Therefore, both $\alpha^{\prime}$ and $1 / N$ corrections go in the same direction. Both types of corrections increase the value of $\gamma$, however, since we are working in the limit where $\lambda \ll N$, the $1 / N$ contributions can be seen as sub-leading ones. ${ }^{4}$

\section{Conclusions}

We have studied type IIB stringy theory corrections to thermalization processes in strongly coupled SYM plasmas. Our results show that both $\alpha^{\prime}$ and $1 / N$ contributions lead to delays in thermalization times for IR modes, while they make slightly faster thermalization time for UV modes, both in comparison with the strong coupling limit, when comparing

\footnotetext{
${ }^{4}$ This holds for geodesic lengths. On the other hand, for Wilson loops the leading $1 / N$ corrections due to world-sheet with handles have been discussed by Drukker and Gross in [39], and they turn to be also additive. Also notice that $\alpha^{\prime}$ corrections to circular Wilson loops due to string fluctuations were discussed by Forste, Ghoshal and Theisen in [40] at zero temperature.
} 
equal temperature or equal energy scenarios. This statement is based on the study of the time evolution of extended geometric probes in the bulk which are connected to specific non-local observables in the boundary field theory plasma.

We find that finite 't Hooft coupling corrections decrease very little the thermalization time of UV modes, while they produce the opposite trend for IR modes, which thermalize slightly later in comparison with their corresponding behavior at the strong coupling limit. Thus, the observed trend is that the leading string theory corrections enhance the difference of thermalization time between UV and IR modes.

In the figures shown we consider the situation where the black hole horizon does not change by the effects of string theory corrections. Equation (2.17) indicates that the equilibrium temperature increases as the 't Hooft coupling decreases from the strong coupling limit. Besides, we also have numerically investigated the case when the equilibrium temperature is kept fixed, and then the black hole radius becomes smaller. In this case our results agree with the pattern indicated in the figures.

Although this approach has no access to the information about coupling corrections to the rate of kinetic energy converted into thermal energy during the heavy-ion collision, it is indeed interesting to study which are the consequences of varying such a conversion rate. We numerically show that if this rate increases, the critical length shifts toward the IR. On the other hand, if it decreases, there is a bound above which there is no crossover and the obtained pattern shows a delay at any scale.

Besides, it has been recently discussed the effect of certain aspects of the thermalization time scale in terms of finite values of the 't Hooft coupling, but from a very different perspective. In particular, in references $[18,19]$ it has been investigated the effect of finite values of the 't Hooft coupling in comparison with the $\lambda \rightarrow \infty$ limit, using a particular version of the holographic thermalization within a quasi-static approach, by looking at spectral densities to infer the thermalization times for UV and IR modes. Recall that spectral densities are obtained from two-point correlation functions of electric currents in the plasma. ${ }^{5}$ Whenever current correlators are studied in the $\mathrm{SU}(N) \mathcal{N}=4$ SYM plasma, it is necessary to introduce an external gauge field in the boundary field theory to be coupled to the current. The currents are to be coupled to a vector fluctuation of the bulk metric, thus turning on a contribution from the Ramond-Ramond five-form field strength in the $\mathcal{O}\left(\alpha^{\prime 3}\right)$ corrections to the type IIB string theory action. These contributions produce very large corrections in SYM plasma observables associated with electric charge transport, such as electrical conductivity, and photo-emission rates. This could be one of the reasons for the difference with respect to our work where no external Abelian gauge field is considered. Another difference is that if one uses non-local observables associated with charged particles in

\footnotetext{
${ }^{5}$ Particularly, for the $\mathrm{SU}(N) \mathcal{N}=4 \mathrm{SYM}$ plasma at thermal equilibrium the current-current correlators, spectral densities, photoemission and lepton pair production rates were firstly obtained in [41]. In order to include the full $\mathcal{O}\left(\alpha^{\prime 3}\right)$ corrections one has to consider not only the effect of the term quartic in the tendimensional Weyl tensor, but also the corrections coming from the Ramond-Ramond 5-form field strength $F_{5}$. These effects can be collected into the $\mathcal{T}$-tensor mentioned above. This leads to a very complicated series of calculations which have been carried out in [42] within the deep inelastic scattering regime, in [43] within the hydrodynamic regime, in [44] for the electrical conductivity of the plasma, in [45, 46] for the photoemission rates in the thermally equilibrated SYM plasma.
} 
the boundary field theory, in order to probe thermalization at a given scale, not only the position of the shell is relevant but also the photon spectrum from the plasma has to be taken into account. In addition, another important difference is that in the approach of $[18,19]$ the idea is to consider a quasi-static shell rather than a dynamical one, and look at certain positions of this shell, which are close to the event horizon of the black hole. From the point of view of the thermalizing plasma this situation corresponds to consider a SYM plasma near the thermal equilibrium. This situation is very different compared with a dynamical holographic thermalization process because in the dynamical case one follows the collapse of the thin shell starting from the boundary of the AdS space, i.e. completely far away from the black hole horizon, and this corresponds to a SYM plasma which is very far from its thermally equilibrated state. This last case seems to be somehow closer to a realistic plasma thermalization process initiated by a heavy ion collision than the quasi-static approach.

It is worth noting that some of the conclusions displayed here could be altered if one considers two-point functions of operators with no large conformal weights. Another crucial ingredient in our work was the assumption of Vaidya as a solution of type IIB supergravity on $A d S_{5} \times S^{5}$ background. Nevertheless, as commented in [30] the pressureless null dust is only understood as an approximation in the field configuration space. For this reason it would be very interesting to consider quantum corrections in the case of more general shells, for instance those discussed in [17] by generalizing the Israel junction conditions in higher derivative gravity theories. By doing so, one should be able to see if the pattern on thermalization times observed here is generic, or it is just a consequence of the degrees of freedom chosen for the shell.

\section{Acknowledgments}

We thank Damián Galante for early collaboration on the subject and for a critical reading of the manuscript. We also thank Robert Myers for valuable discussions concerning entanglement entropy in higher derivative gravity, and to Carlos Núñez and Guillermo Silva for interesting discussions about Wilson loops. This work has been supported by CONICET, the Consejo Nacional de Investigaciones Científicas y Técnicas of Argentina, and the ANPCyT-FONCyT Grant PICT-2007-00849. The work of M.S. has also been supported by the CONICET Grant PIP-2010-0396.

\section{References}

[1] J. Casalderrey-Solana, H. Liu, D. Mateos, K. Rajagopal and U.A. Wiedemann, Gauge/string duality, hot QCD and heavy ion collisions, arXiv:1101.0618 [INSPIRE].

[2] J. Abajo-Arrastia, J. Aparicio and E. Lopez, Holographic evolution of entanglement entropy, JHEP 11 (2010) 149 [arXiv:1006.4090] [INSPIRE].

[3] T. Albash and C.V. Johnson, Evolution of holographic entanglement entropy after thermal and electromagnetic quenches, New J. Phys. 13 (2011) 045017 [arXiv: 1008.3027] [InSPIRE].

[4] H. Ebrahim and M. Headrick, Instantaneous thermalization in holographic plasmas,

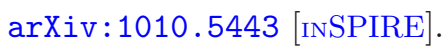


[5] V. Balasubramanian et al., Thermalization of strongly coupled field theories, Phys. Rev. Lett. 106 (2011) 191601 [arXiv:1012.4753] [INSPIRE].

[6] V. Balasubramanian et al., Holographic thermalization, Phys. Rev. D 84 (2011) 026010 [arXiv:1103.2683] [INSPIRE].

[7] D. Garfinkle and L.A. Pando Zayas, Rapid thermalization in field theory from gravitational collapse, Phys. Rev. D 84 (2011) 066006 [arXiv:1106.2339] [INSPIRE].

[8] J. Aparicio and E. Lopez, Evolution of two-point functions from holography, JHEP 12 (2011) 082 [arXiv:1109.3571] [INSPIRE].

[9] A. Allais and E. Tonni, Holographic evolution of the mutual information, JHEP 01 (2012) 102 [arXiv:1110.1607] [InSPIRE].

[10] V. Keranen, E. Keski-Vakkuri and L. Thorlacius, Thermalization and entanglement following a non-relativistic holographic quench, Phys. Rev. D 85 (2012) 026005 [arXiv:1110.5035] [INSPIRE].

[11] D. Garfinkle, L.A. Pando Zayas and D. Reichmann, On field theory thermalization from gravitational collapse, JHEP 02 (2012) 119 [arXiv:1110.5823] [INSPIRE].

[12] S.R. Das, Holographic quantum quench, J. Phys. Conf. Ser. 343 (2012) 012027 [arXiv: 1111.7275$]$ [INSPIRE].

[13] V.E. Hubeny, Extremal surfaces as bulk probes in AdS/CFT, JHEP 07 (2012) 093 [arXiv: 1203.1044] [INSPIRE].

[14] D. Galante and M. Schvellinger, Thermalization with a chemical potential from AdS spaces, JHEP 07 (2012) 096 [arXiv:1205.1548] [INSPIRE].

[15] E. Caceres and A. Kundu, Holographic thermalization with chemical potential, JHEP 09 (2012) 055 [arXiv: 1205.2354] [INSPIRE].

[16] B. Wu, On holographic thermalization and gravitational collapse of massless scalar fields, JHEP 10 (2012) 133 [arXiv: 1208.1393] [INSPIRE].

[17] W. Baron, D. Galante and M. Schvellinger, Dynamics of holographic thermalization, JHEP 03 (2013) 070 [arXiv: 1212.5234] [INSPIRE].

[18] D. Steineder, S.A. Stricker and A. Vuorinen, Holographic thermalization at intermediate coupling, Phys. Rev. Lett. 110 (2013) 101601 [arXiv:1209.0291] [INSPIRE].

[19] D. Steineder, S.A. Stricker and A. Vuorinen, Probing the pattern of holographic thermalization with photons, JHEP 07 (2013) 014 [arXiv: 1304.3404] [INSPIRE].

[20] M.B. Green and C. Stahn, D3-branes on the Coulomb branch and instantons, JHEP 09 (2003) 052 [hep-th/0308061] [INSPIRE].

[21] S. de Haro, A. Sinkovics and K. Skenderis, On a supersymmetric completion of the $R^{4}$ term in IIB supergravity, Phys. Rev. D 67 (2003) 084010 [hep-th/0210080] [INSPIRE].

[22] M.F. Paulos, Higher derivative terms including the Ramond-Ramond five-form, JHEP 10 (2008) 047 [arXiv:0804.0763] [INSPIRE].

[23] R.C. Myers, M.F. Paulos and A. Sinha, Quantum corrections to $\eta / s$, Phys. Rev. D 79 (2009) 041901 [arXiv:0806.2156] [inSPIRE].

[24] M.B. Green and M. Gutperle, Effects of D instantons, Nucl. Phys. B 498 (1997) 195 [hep-th/9701093] [INSPIRE]. 
[25] T. Banks and M.B. Green, Nonperturbative effects in AdS in five-dimensions $\times S^{5}$ string theory and D = 4 SUSY Yang-Mills, JHEP 05 (1998) 002 [hep-th/9804170] [INSPIRE].

[26] M.B. Green, K. Peeters and C. Stahn, Superfield integrals in high dimensions, JHEP 08 (2005) 093 [hep-th/0506161] [INSPIRE].

[27] S.S. Gubser, I.R. Klebanov and A.A. Tseytlin, Coupling constant dependence in the thermodynamics of $N=4$ supersymmetric Yang-Mills theory, Nucl. Phys. B 534 (1998) 202 [hep-th/9805156] [INSPIRE].

[28] J. Pawełczyk and S. Theisen, $A d S_{5} \times S^{5}$ black hole metric at $\mathcal{O}\left(\alpha^{\prime 3}\right)$, JHEP 09 (1998) 010 [hep-th/9808126] [INSPIRE].

[29] S. de Haro, A. Sinkovics and K. Skenderis, On $\alpha^{\prime}$ corrections to D-brane solutions, Phys. Rev. D 68 (2003) 066001 [hep-th/0302136] [INSPIRE].

[30] S. Bhattacharyya and S. Minwalla, Weak field black hole formation in asymptotically AdS spacetimes, JHEP 09 (2009) 034 [arXiv:0904.0464] [INSPIRE].

[31] V. Balasubramanian and S.F. Ross, Holographic particle detection, Phys. Rev. D 61 (2000) 044007 [hep-th/9906226] [INSPIRE].

[32] J.M. Maldacena, Wilson loops in large-N field theories, Phys. Rev. Lett. 80 (1998) 4859 [hep-th/9803002] [INSPIRE].

[33] S. Ryu and T. Takayanagi, Holographic derivation of entanglement entropy from AdS/CFT, Phys. Rev. Lett. 96 (2006) 181602 [hep-th/0603001] [INSPIRE].

[34] V.E. Hubeny and M. Rangamani, Holographic entanglement entropy for disconnected regions, JHEP 03 (2008) 006 [arXiv:0711.4118] [INSPIRE].

[35] E. Witten, Anti-de Sitter space and holography, Adv. Theor. Math. Phys. 2 (1998) 253 [hep-th/9802150] [INSPIRE].

[36] L.-Y. Hung, R.C. Myers and M. Smolkin, On holographic entanglement entropy and higher curvature gravity, JHEP 04 (2011) 025 [arXiv:1101.5813] [INSPIRE].

[37] H. Casini, M. Huerta and R.C. Myers, Towards a derivation of holographic entanglement entropy, JHEP 05 (2011) 036 [arXiv:1102.0440] [INSPIRE].

[38] D.A. Galante and R.C. Myers, Holographic Rényi entropies at finite coupling, arXiv: 1305.7191 [INSPIRE].

[39] N. Drukker and D.J. Gross, An exact prediction of $N=4$ SUSYM theory for string theory, J. Math. Phys. 42 (2001) 2896 [hep-th/0010274] [INSPIRE].

[40] S. Förste, D. Ghoshal and S. Theisen, Stringy corrections to the Wilson loop in $N=4$ super Yang-Mills theory, JHEP 08 (1999) 013 [hep-th/9903042] [INSPIRE].

[41] S. Caron-Huot, P. Kovtun, G.D. Moore, A. Starinets and L.G. Yaffe, Photon and dilepton production in supersymmetric Yang-Mills plasma, JHEP 12 (2006) 015 [hep-th/0607237] [INSPIRE].

[42] B. Hassanain and M. Schvellinger, Holographic current correlators at finite coupling and scattering off a supersymmetric plasma, JHEP 04 (2010) 012 [arXiv:0912.4704] [INSPIRE].

[43] B. Hassanain and M. Schvellinger, Holographic current correlators at finite coupling and scattering off a supersymmetric plasma, JHEP 04 (2010) 012 [arXiv:0912.4704] [INSPIRE]. 
[44] B. Hassanain and M. Schvellinger, Towards 't Hooft parameter corrections to charge transport in strongly-coupled plasma, JHEP 10 (2010) 068 [arXiv: 1006.5480] [INSPIRE].

[45] B. Hassanain and M. Schvellinger, Diagnostics of plasma photoemission at strong coupling, Phys. Rev. D 85 (2012) 086007 [arXiv:1110.0526] [INSPIRE].

[46] B. Hassanain and M. Schvellinger, Plasma photoemission from string theory, JHEP 12 (2012) 095 [arXiv: 1209.0427] [INSPIRE]. 